\title{
MedienPädagogik
}

Zeitschrift für Theorie und Praxis der Medienbildung

Themenheft Nr. 28: Tagungsband: Bildung gemeinsam verändern: Diskussionsbeiträge und Impulse aus Forschung und Praxis. Herausgegeben von David Meinhard, Valentin Dander, Andrea Gumpert, Christoph Rensing, Klaus Rummler und Timo van Treeck.

\section{Förderung beruflicher Handlungskompetenz durch reflektiertes Erfahrungslernen mit digitalen Medien}

Jan Hellriegel

\begin{abstract}
Zusammenfassung
Eine gelingende Entwicklung beruflicher Handlungsfähigkeit in der dualen Berufsausbildung ist auf eine Kooperation von Lehrenden, Ausbildenden und Auszubildenden angewiesen. Häufig scheitert diese Zusammenarbeit jedoch an den spezifischen Lernkulturen und der räumlichen Trennung der verschiedenen Lernorte. Das BMBF-geförderte Forschungsprojekt KOLA: «Kompetenzorientiertes Lernen im Arbeitsprozess mit digitalen Medien» greift diese Problematik auf und verfolgt das Ziel, mithilfe digitaler Medien eine Lernortkooperation zu befördern. Erprobt wird das entwickelte System, bestehend aus einer App und einer Browser-basierten Plattform, in saarländischen Ausbildungsstätten des Elektrohandwerks im Untersuchungszeitraum von Oktober 2014 bis September 2017. Im Rahmen dieses Projektes wird ein lerntheoretischer Ansatz verfolgt, der sich im Kontext von Konstruktivismus und Pragmatismus verorten lässt. Der vorliegende Beitrag fokussiert dabei das didaktische Konzept des Projektes, welches einerseits die didaktischen Grundannahmen und andererseits das hieraus abgeleitete Nutzungsszenario darstellt.
\end{abstract}

Promoting professional management skills through experience-based learning and reflection with digital media

\begin{abstract}
The successful development of professional skills and agency within the German vocational education and training system relies on cooperation between teachers, trainers, and apprentices. However, this cooperation often fails because of the specific learning cultures and the spatial separation of the various places of learning. The BMBFsponsored research project KOLA (competence oriented learning in working process with digital media) addresses this problem with the aim of promoting cooperation between learning sites through the use of digital media. A system that consists of an app and a browser-based platform is being developed and tested in several electrician training companies during an evaluation period from October 2014 to September 2017.

The underlying framework of this project is a learning theory approach that has its foundation in constructivism and pragmatism. The focus of this paper is on the project's didactic concept which presents the basic didactic assumptions as well as the deduced usage scenarios.
\end{abstract}

Hellriegel, Jan. 2017. «Förderung beruflicher Handlungskompetenz durch reflektiertes Erfahrungslernen mit digitalen Medien». MedienPädagogik28, (27. Februar), 66-73. https://doi.org/10.21240/mpaed/28/2017.02.27.X. 


\section{Ausgangslage und Zielsetzung}

Im Laufe der letzten Jahrzehnte hat die Arbeitswelt eine zunehmende Dynamisierung erfahren (vgl. Bünnagel 2012, 11). Während früher Arbeitsplätze ein Leben lang garantiert wurden und sich Arbeitsabläufe kaum gewandelt haben, sind Unternehmen heute einem stetigen Wandel unterworfen. Im Zuge dieser Entwicklungen scheint eine Vermittlung von reinen Fachinhalten an Auszubildende wenig zielführend. Daher fokussiert die duale Berufsausbildung heute mehr denn je eine reflektierte Kompetenzentwicklung (vgl. Riedl 2011,30), welche es Auszubildenden ermöglichen soll, an den Wandel anschlussfähig zu bleiben und zunehmend selbstbestimmt handeln zu können. Kompetenzorientierung ist dabei kein neues Thema. Sowohl im wissenschaftlichen Diskurs als auch in den konkreten Rahmenlehrplänen für die Ausbildungsberufe lässt sich eine Ausrichtung hierauf feststellen (vgl. KMK 2003, 4). Dabei bleibt jedoch fraglich, ob diese strukturellen Zielvorstellungen auch auf personeller Ebene standhalten.

Um den Auszubildenden eine reflektierte Kompetenzentwicklung ermöglichen zu können, sind die beteiligten Lernorte der dualen Berufsausbildung auf eine gemeinsame Kommunikation und Kooperation angewiesen. Die Lehr- und Lernkultur in den Ausbildungsbetrieben, Berufsschulen und den Stätten zur überbetrieblichen Lehrlingsunterweisung (ÜLUs) ist jedoch sehr unterschiedlich, hinzu kommt eine räumliche Trennung der Lernorte (vgl. Arnold und Gonon 2006, 103f.). Diese Faktoren erschweren die gewünschte Zusammenarbeit, was zur Folge haben kann, dass der Theorie-Praxis-Transfer bei den Auszubildenden misslingt (vgl. Pferdt und Kremer 2010, 295).

Diese Annahmen decken sich weitestgehend mit den Ergebnissen einer Anforderungsanalyse, welche im Rahmen des Projektes durchgeführt wurde. In der Erhebung wurden teilnehmende Beobachtungen und leitfadengestützte Interviews mit Berufsschullehrerinnen und Berufsschullehrern, dem Ausbildungspersonal und Auszubildenden einer Berufsschulklasse im Bereich des Elektrohandwerks durchgeführt. Die Ergebnisse wurden angelehnt an eine qualitative Inhaltsanalyse nach Mayring ausgewertet (vgl. Mayring 2015). Dabei weisen Aussagen der Akteurinnen und Akteure darauf hin, dass es Auszubildenden häufig schwer fällt, Bezüge zwischen den theoretischen Inhalten aus der Schule und der praktischen Arbeit in den Ausbildungsbetrieben herzustellen. Lehrende betonen, dass sie selbst zwar ein grundsätzliches Interesse an den bearbeiteten Aufträgen der Auszubildenden aus den Ausbildungsbetrieben haben, sich aber nicht explizit beim Ausbildungspersonal danach erkundigen und das Berichtsheft kaum Einblick in andere Lernorte bietet (vgl. Hellriegel et al. 2015, 68f.).

Aufbauend auf diesen Problemdarstellungen verfolgt das Forschungsprojekt KOLA das Ziel, eine Kooperation der Lernorte zu befördern und die Entwicklung der beruflichen Handlungskompetenz bei den Auszubildenden zu stärken. Dabei spielt der 
Einsatz digitaler Medien eine zentrale Rolle, da diese für eine räumlich und zeitlich entkoppelte Zusammenarbeit genutzt werden können und dabei neue Handlungssphären eröffnen, welche an bisherige reale Handlungen anknüpfen (vgl. Unger 2010, 99). Im Rahmen des Projektes wurde ein didaktisches Konzept entwickelt, welches als Ausgangspunkt für die Konzeption einer App und einer Online-Plattform diente. Mithilfe der App können Auszubildende ihre Arbeitshandlungen und Erfahrungen digital erfassen. Die Online-Plattform ermöglicht es den Ausbildenden und Lehrenden der beteiligten Lernorte auf diese Einträge Bezug zu nehmen, im eigenen Lernort aufzugreifen und zu thematisieren.

\section{Didaktische Grundannahmen}

Das didaktische Konzept des KOLA-Projektes stellt im Wesentlichen Bezüge zu den lerntheoretischen Grundannahmen des Konstruktivismus und des Pragmatismus her. Diese beiden Theorien werden als sich ergänzende Ansätze aufgefasst, die eine berufliche Handlungskompetenz mithilfe digitaler Medien fördern können. Abbildung 1 stellt diesen Gesamtzusammenhang dar.

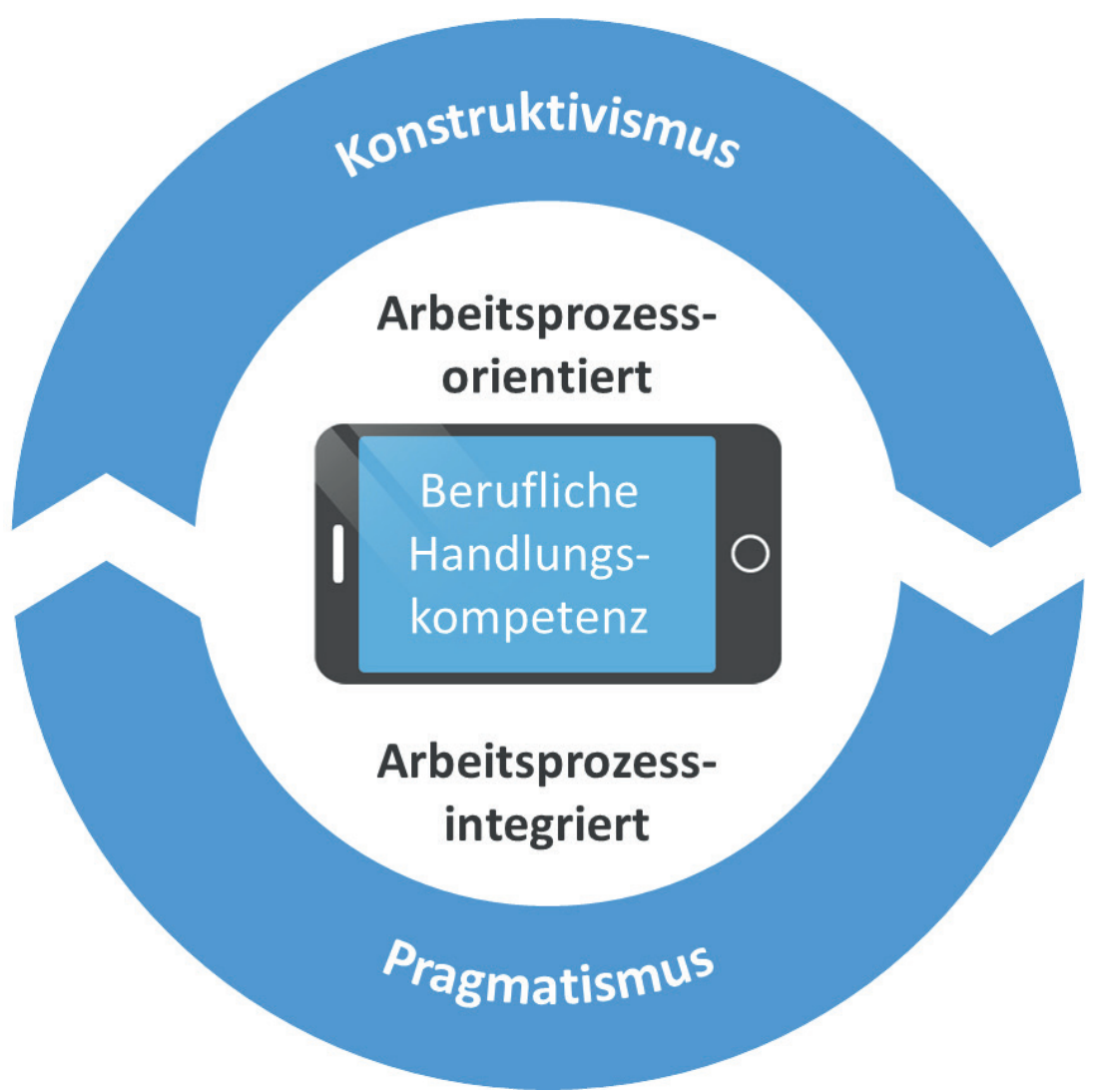

Abb. 1.: Lerntheoretische Grundannahmen im Projekt KOLA (eigene Darstellung). 
Das Projekt knüpft dabei an das dreiteilige Modell der Berufskompetenz nach Schelten an. Dabei sind neben Fachkompetenzen auch Personal- und Sozialkompetenzen von Bedeutung (vgl. Schelten 2010, 172). Sozialkompetenzen beziehen sich dabei auf den Umgang mit anderen Personen, Personalkompetenzen auf eine gelingende Identitätsentwicklung (vgl. Riedl 2011, 39). Im Zuge einer dynamisierten Arbeitswelt gewinnen jedoch auch zunehmend sogenannte Metakompetenzen an Bedeutung. Diese werden als Basis für eine selbstkritische und reflexive Grundhaltung benötigt, um in einer von stetigen Veränderungen geprägten Arbeitswelt selbstbestimmt in neuen Situationen handeln zu können. Erpenbeck bezeichnet diese als «Bedingungen der Möglichkeit unserer Selbstorganisation» (Erpenbeck 1995, 8).

Um solch eine facettenreiche Entwicklung gewährleisten zu können, wird das Ziel verfolgt, sowohl ein arbeitsprozessintegriertes als auch ein arbeitsprozessorientiertes Lernen zu befördern. Ansätze zur Arbeitsprozessorientierung finden sich dabei vor allem im Konstruktivismus. Konstruktivistische Ansätze folgen der Grundannahme, dass sich eine äussere Realität nicht objektiv und ungefiltert erfassen lässt (vgl. Reich 2012, 75). Lernprozesse sind immer an sozio-kulturelle Hintergründe gebunden. Infolgedessen konstruiert jede Person ein eigenes Bild von Realität, welches von anderen Realitätsauffassungen abweicht. Dieser Vorstellung folgend, scheinen Methoden wie die des Frontalunterrichts wenig zielführend, da Lehrende Wissen nie vollends vorhersehbar in den Lernenden erzeugen können. Was dem Frontalunterricht fehlt, sind aktive und kreative Prozesse, die eine eigene Teilhabe und Gestaltung beinhalten. Konstruktivistische Ansätze betonen diese aktive Seite (vgl. von Glasersfeld 2002, 220) und plädieren für ein fallorientiertes Lernen, welches sich an Situationen beruflicher Praxis orientiert. Fokussiert werden dabei besonders problembezogene Fälle, die anhand komplexer Strukturen eigene Konstruktionen ermöglichen (vgl. Kerres und de Witt 2004, 4).

Das KOLA-Projekt distanziert sich dabei allerdings von einem Standpunkt, der Instruktion völlig ablehnt. Im Bereich der beruflichen Ausbildung wird eher ein Mittelweg zwischen Unterrichtsformaten gewählt, bei denen zwischen einer lernenden- und lehrendenzentrierten Sichtweise situationsabhängig gewechselt werden kann. Das didaktische Konzept verfolgt daher einen Ansatz des gemässigten Konstruktivismus. Bezüglich der Zielsetzung einer Arbeitsprozessintegration lassen sich zudem Bezüge zum Pragmatismus in Anlehnung an den Ansatz von John Dewey herstellen. Der Pragmatismus versteht Lernen als einen aktiven Vorgang und richtet sich stark an den Erfahrungen der Akteurinnen und Akteure aus (vgl. Reich 2012, 71). Dewey legt dabei Wert auf eine Auseinandersetzung mit realen Praxissituationen, aus denen sich Problemsituationen ableiten lassen und die zur Reflexion genutzt werden können (vgl. Dewey 2000 [1916], 170). Der Pragmatismus scheint als theoretische Grundlage für das Projekt insofern geeignet, da er reale Arbeitssituationen in den Vordergrund stellt und somit eine Praxisintegration einer Praxisorientierung vorzieht. Ebenso 
plädiert der Pragmatismus dafür, dass Lernende selbst verschiedene Blickwinkel einnehmen und eigene Handlungen aus mehreren Perspektiven betrachten (vgl. Kerres und de Witt 2004, 12f.).

\section{Systemnutzung}

Nachfolgend sollen die praktischen Bezüge auf der Ebene der Systemnutzung dargestellt werden, welche sich aus den Ergebnissen der Anforderungserhebung und den didaktischen Annahmen bezüglich einer Arbeitsprozessintegration und Arbeitsprozessorientierung ableiten (vgl. Abb. 2).

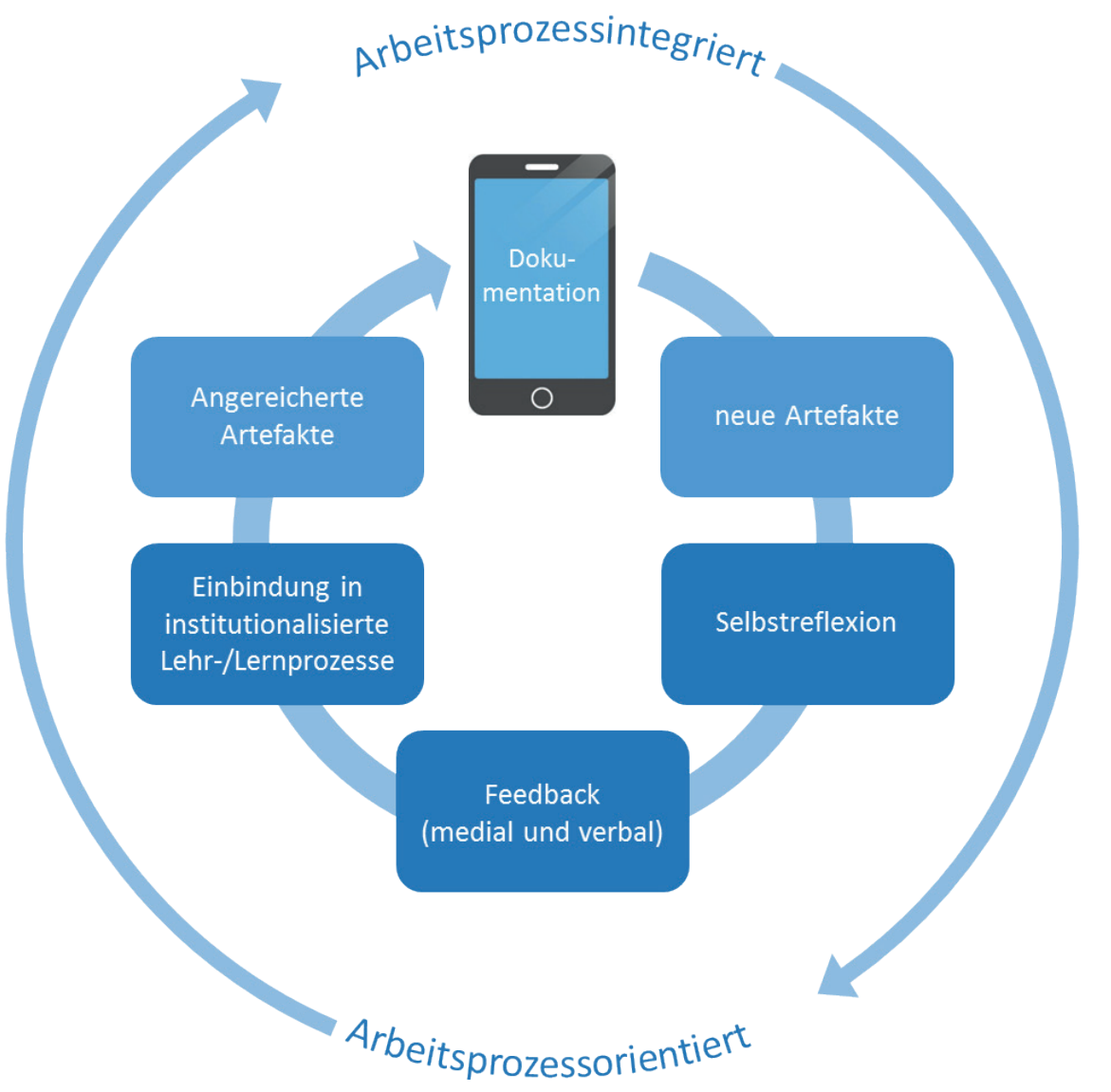

Abb. 2.: Systemnutzung im Projekt KOLA (eigene Darstellung).

Die Arbeitsprozessintegration soll dadurch gewährleistet werden, dass die Auszubildenden in allen drei Lernorten durchgeführte Arbeiten, vermittelte Themen und gesammelte Erfahrungen medial erfassen. Damit dies gelingen kann, steht den Auszubildenden eine in Teilschritte gegliederte Strukturvorlage im System zur Verfügung, 
die es ihnen erleichtert, komplexere Arbeitsprozesse in einzelne Teilschritte zu zerlegen (siehe Abb. 3). Nachdem die Handlungen erfasst wurden, haben die Auszubildenden die Möglichkeit anhand von im System hinterlegten Reflexionsfragen eigene Handlungen kritisch zu bewerten und aus einem anderen Blickwinkel zu beleuchten.
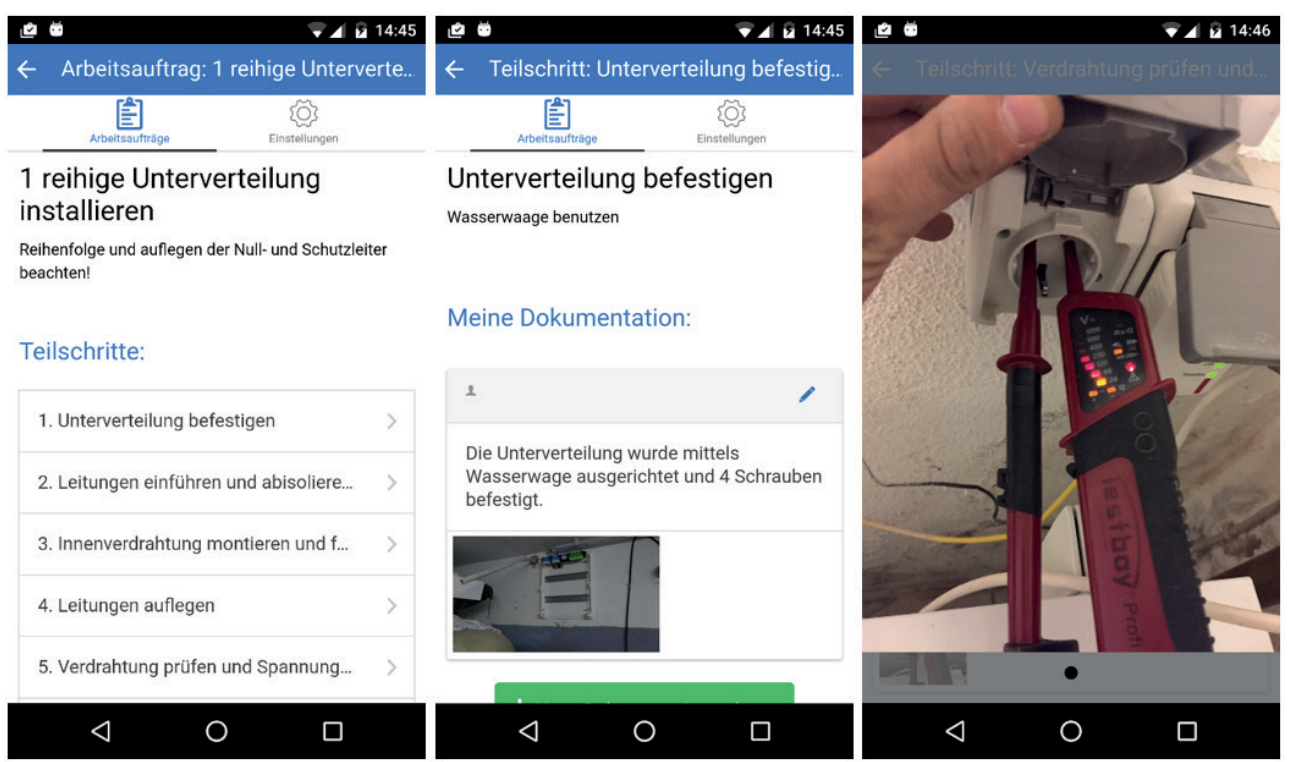

Abb. 3.: Übersicht einer durchgeführten Dokumentation (App-Screenshots).

Eine Arbeitsprozessorientierung wird vor allem durch eine nachträgliche Kommunikation angestrebt, welche sich an die Dokumentationen und Selbstreflexionen anschliesst. Ausbildende und Lehrende haben die Möglichkeit, die Einträge der Auszubildenden zu betrachten und diese zum Anlass für Feedbackgespräche zu nehmen oder in institutionalisierten Lehr-/Lernprozessen aufzugreifen. Ausbildende, welche Auszubildende nicht auf den Baustellen begleiten, können beispielsweise nach der morgendlichen Einsatzplanung anhand der Fotos, Textbeiträge und den beantworteten Reflexionsfragen mit den Auszubildenden bereden, wie gut sie mit den Aufträgen zurechtkamen und wo noch Nachholbedarf besteht. Lehrende können die Dokumentationen im Sinne von Praxisbeispielen zu einem freigewählten Zeitpunkt im Unterricht thematisieren. So kann eine Dokumentation mit Mess- und Prüfergebnissen einer Elektroinstallation in der Berufsschule aufgegriffen werden, wenn das Thema im Lehrplan vorgesehen ist. Die Dokumentationen können von den einzelnen Akteurinnen und Akteuren im System kommentiert werden und dienen den Auszubildenden dann wieder als Vorlage zum Nachlesen, beispielsweise für kommende Arbeitsaufgaben, die an Komplexität zunehmen oder etwa für Prüfungsvorbereitungen. 


\section{Fazit}

Um Auszubildenden eine reflektierte Handlungsfähigkeit für das spätere Berufsleben ermöglichen zu können, muss an deren Erfahrungen und Handlungen in der beruflichen Ausbildung angeknüpft werden. Mithilfe des entwickelten Systems strebt das KOLA-Projekt ein Setting an, in dem reale Praxissituationen systematisch aufgegriffen, reflektiert und analysiert werden und in einen Kontext mit theoretischen Inhalten gesetzt werden, um damit einen Theorie-Praxis-Transfer zu ermöglichen.

\section{Literatur}

Arnold, Rolf, und Philipp Gonon. 2006. Einführung in die Berufspädagogik. Opladen und Bloomfield Hills: Barbara Budrich.

Bünnagel, Werner. 2012. Selbstorganisiertes Lernen im Unternehmen. Motivation freisetzen, Potenziale entfalten, Zukunft sichern. Wiesbaden: Gabler.

Dewey, John. 2000. Demokratie und Erziehung. [1916] Weinheim: Beltz.

Erpenbeck, John. 1995. «Metakompetenzen und Selbstorganisation.»In Metakompetenzen und Kompetenzentwicklung. QUEM-report, Schriften zur beruflichen Weiterbildung, herausgegeben von Arbeitsgemeinschaft Betriebliche Weiterbildungsforschung e. V., 5-14. Berlin: ESM.

Glasersfeld, Ernst von. 2002. «Was heißt Lernen aus konstruktivistischer Sicht.»In Unterricht aus konstruktivistischer Sicht: Die Welten in den Köpfen der Kinder, herausgegeben von Reinhard Voss, 214-223. Weinheim [u.a.]: Beltz.

Hellriegel, Jan, Regina Osranek, Thomas Prescher, Christoph Rensing, und Harald Weber. 2015: «Herausforderungen und Konsequenzen für die Konzeption eines digitalen Lernraumes in der beruflichen Erstausbildung zur Förderung der Lernortkooperation.» In Proceedings of DeLFI Workshops 2015 co-located with 13th e-Learning Conference of the German Computer Society (DeLFI 2015), herausgegeben von Sabine Rathmayer und Hans Pongratz, 65-71. München.

Kerres, Michael, und Claudia de Witt. 2004. «Pragmatismus als theoretische Grundlage zur Konzeption von eLearning. Grundlagen und Beispiele.» In Handlungsorientiertes Lernen und eLearning. Grundlagen und Beispiele, herausgegeben von Dietmar Treichel und Horst Mayer, 77-100. München und Wien: Oldenbourg Wissenschaftsverlag.

KMK (Kultusministerkonferenz). 2003. Rahmenlehrplan für den Ausbildungsberuf Elektroniker/ Elektronikerin. http://www.kmk.org/fileadmin/pdf/Bildung/BeruflicheBildung/rlp/elektroniker.pdf.

Mayring, Philipp. 2015. Qualitative Inhaltsanalyse: Grundlagen und Techniken. Weinheim und Basel: Beltz. 
Pferdt, Frederik, und H.-Hugo Kremer. 2010. «Berufliches Lernen mit Web 2.0 - Medienkompetenz und berufliche Handlungskompetenz im Duell?» In Jahrbuch Medienpädagogik 8. Medienkompetenz und Web 2.0, herausgegeben von Bardo Herzig, Dorothee M. Meister, Heinz Moser und Horst Niesyto, 289-308. Wiesbaden: VS Verlag für Sozialwissenschaften.

Reich, Kersten. 2012. Konstruktivistische Didaktik: Das Lehr- und Studienbuch mit Online-Methodenpool. Weinheim: Beltz.

Riedl, Alfred. 2011. Didaktik der beruflichen Bildung. Stuttgart: Franz Steiner.

Schelten, Andreas. 2010. Einführung in die Berufspädagogik. Stuttgart: Franz Steiner.

Unger, Alexander. 2010. «Virtuelle Räume und die Hybridisierung der Alltagswelt.» In Neue digitale Kultur- und Bildungsräume, herausgegeben von Petra Grell, Winfried Marotzki und Heidi Schelhowe, 99-118. Wiesbaden: VS Verlag für Sozialwissenschaften.

\section{Abbildungen}

Abb. 1.: Lerntheoretische Grundannahmen im Projekt KOLA (eigene Darstellung).

Abb. 2.: Systemnutzung im Projekt KOLA (eigene Darstellung).

Abb. 3.: Übersicht einer durchgeführten Dokumentation (App-Screenshots). 